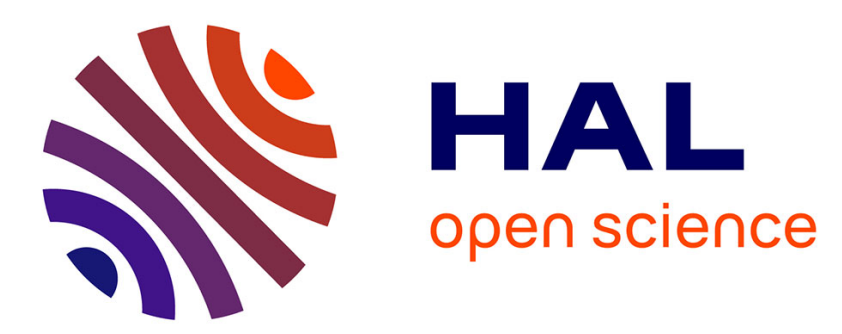

\title{
A simple and accurate new method to measure specific heat at arbitrary temperature (quotient method)
}

\author{
R. Marx
}

\section{To cite this version:}

R. Marx. A simple and accurate new method to measure specific heat at arbitrary temperature (quotient method). Revue de Physique Appliquée, 1978, 13 (6), pp.298-303. 10.1051/rphysap:01978001306029800 . jpa-00244453

\section{HAL Id: jpa-00244453 https://hal.science/jpa-00244453}

Submitted on 1 Jan 1978

HAL is a multi-disciplinary open access archive for the deposit and dissemination of scientific research documents, whether they are published or not. The documents may come from teaching and research institutions in France or abroad, or from public or private research centers.
L'archive ouverte pluridisciplinaire HAL, est destinée au dépôt et à la diffusion de documents scientifiques de niveau recherche, publiés ou non, émanant des établissements d'enseignement et de recherche français ou étrangers, des laboratoires publics ou privés. 
Classification

Physics Abstracts

$05.70-06.60-07.20 \mathrm{~F}$

\title{
A SIMPLE AND ACCURATE NEW METHOD TO MEASURE SPECIFIC HEAT AT ARBITRARY TEMPERATURE (QUOTIENT METHOD) (*)
}

\author{
R. MARX \\ Physikalisches Institut der Universität zu Köln, Germany (**) \\ Reçu le 27 juin 1977, révisé le 20 février 1978, accepté le 24 février 1978)
}

\begin{abstract}
Résumé. - On décrit une nouvelle méthode qui permet en principe la mesure de la chaleur spécifique à toutes les températures sur des échantillons relativement petits. Voici les avantages principaux comparés aux méthodes conventionnelles : le calcul de la chaleur spécifique inconnue est très simple (un calculateur électronique n'est pas nécessaire), on ne doit pas connaître la quantité d'énergie avec laquelle l'échantillon a été chauffé, la tension de sortie du couple thermoélectrique peut être mesurée avec des unités arbitraires et l'influence de l'environnement est moins critique que pour des méthodes conventionnelles. La construction de l'appareil ne demande que très peu de temps et il n'est pas nécessaire d'automatiser la méthode.
\end{abstract}

\begin{abstract}
A new method is described which allows the measurement of specific heat in principle at arbitrary temperature and of relatively small samples. The main advantages compared with conventional methods are : The calculation of the unknown specific heat from the raw data is very simple (no computer is needed), the heater input needs not be known, the thermocouple outputs may be measured in arbitrary units, and the coupling to the surroundings is much less crucial than it is for conventional methods. The building of the apparatus can be completed within a few month and it is very easy to automate the measuring process.
\end{abstract}

1. Introduction. - The purpose of the equipment described here is to measure the specific heat, $C_{p}$, of solids from liquid helium temperature up to room temperature, although the usefulness of the method is not restricted to this special temperature range.

We decided to build an apparatus whose development and construction should require as little time as possible. In addition the automating of the measuring process should be easy because we want to measure a lot of amples in a short time. For these reasons we started to develop a quotient method which will be described in the next chapters.

2. Principles of method used and relation to other methods. - The common difficulty of all absolute methods to measure specific heat consists in the precise determination of the heat delivered to the sample and in the accurate measurement of the corresponding temperature change. To determine the specific heat correctly, therefore, the temperature before and after a heat pulse must be measured to a high accuracy in the presence of temperature drifts.

At high temperatures differences in temperature between the adiabatic shield and the sample can cause

(*) Work performed within the research program of the Sonderforschungsbereich 125 Aachen/Jülich/Köln.

$(* *)$ New address : Labor für Tieftemperaturphysik der Gesamthochschule Duisburg, 4100 Duisburg, Germany. an unbalanced heat leak due to radiation and thus falsify the results. It is believed that the uncertainty in the evaluation of this heat leak and of the heat leak due to wires connecting the sample and the surroundings are the principal sources of error in a large fraction of measurements for accurate calorimetry. It is an old idea in physics to reduce the influence of errors of the described type by replacing the absolute measurement by a relative measurement so that the error will be reduced or even removed. According to this idea R. W. Jones et al. [1] tried to overcome the difficulties and measuring errors of the absolute measuring methods by proposing a Differential Calorimetry Technique. They built a twin sample configuration which consisted of two samples, one with known and one with unknown specific heat and connected these two samples by a thermal resistor. The thermal coupling between the two samples was strong compared with the coupling of the whole arrangement to the surroundings. Both samples were heated at the same time and if the heater input was manipulated such that there appeared no temperature difference between the two samples during and after the heating then the ratio of the heat capacities was equal to the ratio of the heater inputs. This idea should work very well if the inner relaxation times of the samples are equal or nearly equal.

In reality, however, this is nearly never the case. To 
cope with the trouble caused by different relaxation times it is necessary to take complicated additional measures which make the handling troublesome and the evaluation of the specific heat rather tedious. The interested reader is referred to the paper of Jones et al. [1] in which the problems are explained in detail.

Therefore, we developed the measuring procedure described below. We seize on the idea of coupling two samples strongly together (Fig. 1) and make the following experiment. First, we send the heater input $\mathbf{H}$ into heater $\mathrm{H}_{1}$. The input must be well defined but needs not be known $\left({ }^{1}\right)$. After heating we wait for new equilibrium of the whole arrangement which will be recovered after about six minutes.

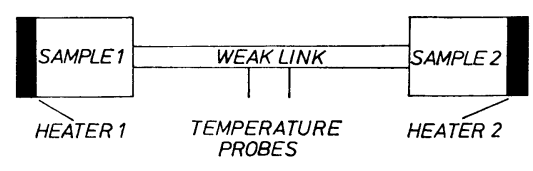

FIG. 1. - Twin arrangement.

Then we repeat the heating but now we send the heater input $\mathbf{H}$ into heater $\mathbf{H}_{2}$. It is important to take exactly the same input $H$. Then we wait for new equilibrium again. During the heating and during the dissipation of heat in both cases we record the heat flux through the weak link as a function of time (Figs. 2 and 3) :

$$
\dot{Q}(x, t)=-\lambda A \frac{\partial T}{\partial x}(x, t)
$$

( $\lambda$ thermal conductivity of the weak link, $A$ conduction crossection, $\partial T / \partial x$ temperature gradient ; the flux $\dot{Q}$ will be independent of $x$, if the diameter of the weak link is independent of $x$ and if the material is homogeneous). Then :

1) heating into $H_{1}$ and dissipating of heat from $S_{1}$ to $\mathrm{S}_{2}$

$$
\int_{0}^{+\infty} \dot{Q}_{1}(t) \mathrm{d} t=Q_{01}=C_{2}\left(T_{\mathrm{f}}-T_{\mathrm{i}}\right)_{1} .
$$

2) heating into $\mathrm{H}_{2}$ and dissipating of heat from $\mathrm{S}_{2}$ to $\mathrm{S}_{1}$

$$
\int_{0}^{+\infty} \dot{Q}_{2}(t) \mathrm{d} t=Q_{02}=C_{1}\left(T_{\mathrm{f}}-T_{\mathrm{i}}\right)_{2}
$$

and, because in both cases the heater inputs are equal, we have

$\left(T_{\mathrm{f}}-T_{\mathrm{i}}\right)_{1}=\left(T_{\mathrm{f}}-T_{\mathrm{i}}\right)_{2}$ for $\left(T_{\mathrm{f}}-T_{\mathrm{i}}\right)_{1,2} \rightarrow 0$

$\left(Q_{01}\right.$ heat dissipated from $\mathrm{S}_{1}$ to $\mathrm{S}_{2}, Q_{02}$ vice versa, $C_{1}$ heat capacity of sample $\mathrm{S}_{1}, C_{2}$ heat capacity of

(1) Order of magnitude of the input 1 joule, heating period about $60 \mathrm{~s}$, weights of sample $S_{1}$ and sample $S_{2}$ about $2 \mathrm{~g}$ (copper and materials with similar Debye temperatures); all data and calculations here and in the following text are referred to $300 \mathrm{~K}$, as far as they are obviously temperature dependent. The situation at $T=300 \mathrm{~K}$ is the most difficult one, at lower temperatures it becomes somewhat more convenient.

REVUE DE PHYSIQUE APPLIQUÉE. - T. 13, N 6, JUIN 1978 sample $\mathrm{S}_{2}, T_{\mathrm{i}}$ equilibrium temperature of the twin arrangement before heating, $T_{\mathrm{f}}$ equilibrium temperature after dissipation of heat, $T_{\mathrm{f}}-T_{\mathrm{i}}$ is about $0.5 \mathrm{~K}$ ). Sample $S_{2}$ should be a material the specific heat of which is well defined and exactly known (e. g. copper). It is an easy task to measure the heat flux through the weak link because this can be done by mounting two temperature probes on to the weak link (for detailed information consult next chapter). If the quantities $Q_{01}$ and $Q_{02}$ are known then it is a straightforward problem to calculate the unknown heat capacity $C_{1}$ :

$$
C_{1}=\eta\left(C_{2}+C_{\mathrm{a} 2}\right)-C_{\mathrm{a} 1}
$$

$C_{\mathrm{a} 1}$ and $C_{\mathrm{a} 2}$ refer to the heat capacities of the addenda of samples 1 and 2 , respectively $\eta=Q_{02} / Q_{01}$. Therefore, only the values of the integrated heat fluxes $Q_{01}$ and $Q_{02}$ and the heat capacities of the reference sample and of the addenda must be determined. Insteed of the integrated heat fluxes it is sufficient to measure two quantities $A_{1}$ and $A_{2}$ in arbitrary units which are proportional to $Q_{01}$ and $Q_{02} . C_{\mathrm{a} 1}$ and $C_{\mathrm{a} 2}$ can be calculated from measurements of two reference samples of the same material with known specific heat but with different masses. Systematic errors (caused by poor vacuum, radiation, conduction through leads etc.) are largely cancelled as is demonstrated by the following error calculation.

$$
\begin{aligned}
& A_{\mathrm{m} 1}=A_{1} \pm \Delta A_{1} \\
& A_{\mathrm{m} 2}=A_{2} \pm \Delta A_{2} .
\end{aligned}
$$

Then (if $\Delta A_{k} \ll A_{k}(k=1,2)$ )

$A_{1} / A_{2} \approx A_{\mathrm{m} 1} / A_{\mathrm{m} 2}\left[1 \pm\left(\Delta A_{1} / A_{\mathrm{m} 1}-\Delta A_{2} / A_{\mathrm{m} 2}\right)\right]$

( $A_{\mathrm{m} k}$ measured quantities, $A_{k}$ true quantities $; k=1,2$ ). It is evident from equation (5) that systematic errors (equal signs of $\Delta A_{1}$ and $\Delta A_{2}$ ) are cancelled to a large extent.

In equation (4), the heat capacities of sample 1 and sample 2 are compared at different temperatures. Because of $T_{\mathrm{f}}-T_{\mathrm{i}}$ small this will cause a negligible error if the specific heats of the samples are only slightly temperature dependent. If necessary there is an easy possibility to avoid this error : plot the quantities $A_{\mathrm{mk}}$ as functions of temperature, connect the dots by a smooth line and divide the one graph by the other. The resulting function is the correct $\eta=\eta(T)$.

3. Construction of the weak link, determination of the heat flux. - We manufactured the weak link out of Au-0.07 at \% Fe (length $16 \mathrm{~mm}, 6$ twisted wires with a diameter of $300 \mu$ each). As temperature probes we spotwelded two chromel wires (diameter $100 \mu$ ) on to the weak link so that the welding point was the place at which the temperature was measured. (Thermocouples as temperature sensors should work very well above $1.5 \mathrm{~K}$. Below $1.5 \mathrm{~K}$ other sensors will be needed). The thermal resistance of the weak link is about $200 \mathrm{~K} / \mathrm{W}$. The coupling of the twin arrange- 
ment to the surroundings (through leads etc.) has a resistance of about $2 \times 10^{5} \mathrm{~K} / \mathrm{W}$. Therefore, the weak link is a strong link between the samples compared with the coupling of the samples to the surroundings. On the other hand the link is weak, because the relaxation time of the twin arrangement $\tau$ (equation (13)) is large compared with the inner relaxation times of the samples $\tau_{\mathrm{i}}^{k}\left(\tau \approx 62 \mathrm{~s} ; \tau_{\mathrm{i}}^{k} \leqslant 5 \mathrm{~s} ; \tau_{\mathrm{i}}^{k}=C_{k} . R_{k}\right.$, $k=1,2$. $R_{k}$ thermal resistances of the samples).

In the following we discuss how to measure the heat flux. According to equation (1) this problem is reduced to measuring the temperature gradient across the weak link ( $\lambda$ and $A$ need not to be known because $\eta$ does not depend on these parameters). Because of the finite distance between the temperature probes we do not measure the differential quotient but only the difference quotient $\Delta T(t) / \Delta x$ which is always smaller than $\mathrm{d} T / \mathrm{d} x$ (Fig. 4). This causes a systematic measuring error and we have to find out on which conditions the error will not exceed a certain limit. Figure 2

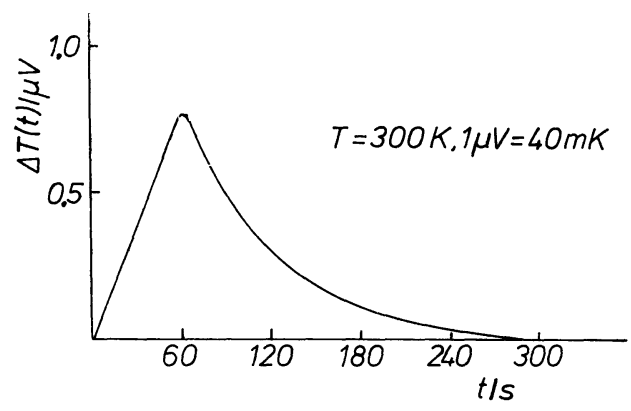

FIG. 2. - Temperature difference $\Delta T(t)$ between the temperature probes as a function of time.

shows the temperature difference $\Delta T$ between the two temperature probes (Fig. 1) as a function of time. During the heating there is an almost linear increase and after the heating an almost exponential decrease of the temperature difference (see equations $(11,12)$ and comment to these equations). To solve the problem on which conditions we can replace $\mathrm{d} T(t) / \mathrm{d} x$ by $\Delta T(t) / \Delta x$ we consider a rod whose length is much larger than its diameter (Fig. 3).

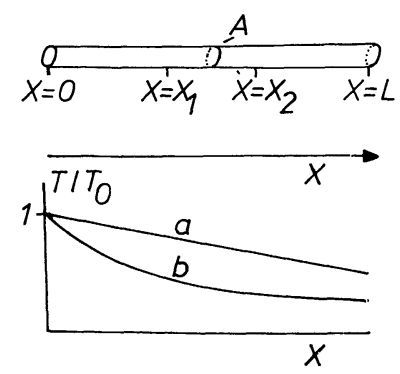

FIG. 3. - Rod representing the weak link and the temperature ratio $T / T_{0}$ along the weak link as a function of distance $x$ in two cases : $a$ ) stationary heat flux and $b$ ) nonstationary heat flux (schematically; $T_{0} \equiv T(x=0)$ ).
The temperature as a function of distance and time is described by the diffusion equation :

$$
\frac{\mathrm{d}^{2} T}{\mathrm{~d} x^{2}}=R^{\prime} C^{\prime} \frac{\mathrm{d} T}{\mathrm{~d} t} \quad T=T(x, t)
$$

$\left(R^{\prime}, C^{\prime}\right.$ are the thermal resistance and the heat capacity per unit length of the rod, respectively). Identifying the weak link with the rod and heating into heater $\mathrm{H}_{1}$ (for example) we get the following temperature along the weak link for the time $t=t_{1}$ (Fig. 3) : Curve a) shows the temperature in case of a stationary heat flux and curve $b$ ) in case of a nonstationary heat flux. The deviation from a linear dependence will increase if $R^{\prime}$ decreases and/or $C^{\prime}$ increases and the deviation will decrease in the opposite case.

Since we want to make a Fourier transformation of the temperature $T=T(t)$, we try for equation (6) the solution

$$
\bar{T}(x, t)=\bar{T}(x) \mathrm{e}^{i \omega t} .
$$

Substituting this ansatz into equation (6), we get

$$
\frac{\mathrm{d}^{2} \bar{T}(x)}{\mathrm{d} x^{2}}=i R^{\prime} C^{\prime} \omega \bar{T}(x)
$$

and therefore

$$
T(x)=|\bar{T}(x)|=T_{0} \exp \left(-\sqrt{\frac{\omega R^{\prime} C^{\prime}}{2}} x\right)
$$

$\left(T_{0} \equiv T(x=0)\right.$; for physical reasons we are interested in the minus sign only).

Now we are able to compare the differential with the difference quotient and get the result

$$
\begin{aligned}
& \begin{array}{r}
\Delta T(t)=T\left(x=x_{1}, t\right)-T\left(x=x_{2}, t\right), \\
\left.\Delta x=x_{2}-x_{1}, 0<x<L\right)
\end{array} \\
& \begin{aligned}
\frac{\Delta T}{\Delta x} \cdot \frac{\mathrm{d} x}{\mathrm{~d} T}=\sqrt{\frac{2}{\omega \cdot \tau_{\mathrm{wl}}}} \times \\
\quad \times\left(1-\exp \left[-\sqrt{\frac{\omega \cdot \tau_{\mathrm{wl}}}{2}} \frac{\Delta x}{L}\right]\right) \cdot \frac{L}{\Delta x}
\end{aligned}
\end{aligned}
$$

$\left(R=R^{\prime} . L, C=C^{\prime} . L\right.$ and $\tau_{\mathrm{wl}}=R . C$ inner relaxation time of the weak link, $\tau_{\mathrm{wl}} \approx 3 \mathrm{~s}$ ). In order to consider the question which frequencies $\omega$ are relevant here we make a Fourier analysis of $\Delta T(t)$. For the time dependence of $\Delta T$ the following simplifying assumptions are valid :

$$
\begin{array}{ll}
\Delta T(t)=b \cdot t & 0 \leqslant t \leqslant t_{\max } \\
\Delta T(t)=\Delta T_{\max } \mathrm{e}^{-t / \tau} & t_{\max } \leqslant t \leqslant t_{\infty}
\end{array}
$$

$\left(\Delta T_{\max }=\Delta T\left(t=t_{\max }\right) ; b \approx 1.7 \mathrm{mK} / \mathrm{s}, t_{\max } \approx 60 \mathrm{~s} ;\right.$ $\tau \approx 62 \mathrm{~s}$; all this data from experiment, see figure 2 ). These equations are appropriate approximations, if the contact resistances between the heaters and the samples are negligible and if $\tau_{\mathrm{i}}^{k} \ll \tau$. Under these assumptions [2]

$$
\tau=\frac{C_{1} \cdot C_{2}}{C_{1}+C_{2}} \cdot R
$$


If we write

$$
\Delta T(\omega)=\int_{-\infty}^{+\infty} \Delta T(t) \mathrm{e}^{-i \omega t} \mathrm{~d} t,
$$

and

$$
\Delta T(t)=\frac{1}{2 \pi} \int_{-\infty}^{+\infty} \Delta T(\omega) \mathrm{e}^{i \omega t} \mathrm{~d} \omega
$$

then in case of equation (11):

$$
\begin{aligned}
\Delta T(\omega)=\frac{2 b}{\omega} t_{\max } & \sin \omega t_{\max }+ \\
+ & i \frac{2 b}{\omega}\left[t_{\max } \cos \omega t_{\max }-\frac{\sin \omega t_{\max }}{\omega}\right]^{(2)}
\end{aligned}
$$

in case of equation (12)

$$
\Delta T(\omega)=\Delta T_{\max } \cdot \tau \frac{1-i \omega \tau}{1+\omega^{2} \tau^{2}} .
$$

A rough calculation shows that essentially those frequencies contribute to $\Delta T(t)$ which satisfy the condition $0 \leqslant \omega \leqslant 2 \omega_{\tau} ; \omega_{\tau}=2 \pi / \tau$. Therefore, we have to consider in (10) only these frequencies. Figure 4 shows the result. If $\Delta x / L=1 / 8$ (see arrow), then $\Delta T / \Delta x . \mathrm{d} x / \mathrm{d} T=0.97$. Consequently, the upper limit of the error we make is about $3 \%$ if we replace the differential by the difference quotient. As a systematic error it will largly be reduced if $\eta$ is calculated (cf. equation (5)). Because of the form of the equations (5) and (2) a more careful and exact analysis of the measuring error represents a serious mathematical problem and has therefore been omitted.

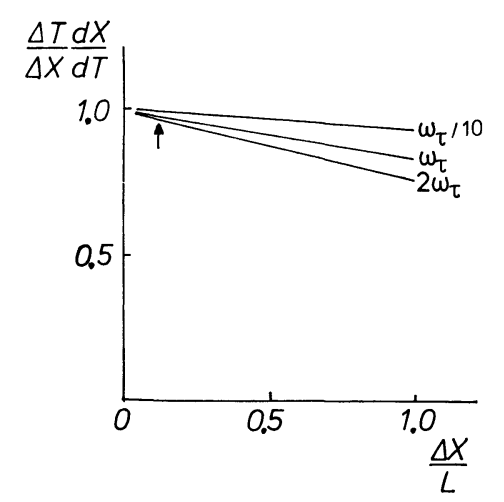

FIG. 4. - Comparison between difference and differential quotient for the frequency range $0 \leqslant \omega \leqslant 2 \omega_{\tau} ; \omega_{\tau}=2 \pi / \tau$.

4. Experimental arrangement, thermometry. 4.1 Cryostat. - We used a simple ${ }^{4} \mathrm{He}$ glass cryostat with an inner diameter of $90 \mathrm{~mm}$. The lowest temperature of the bath we reached was $1.4 \mathrm{~K}$. Its volume was about 6 litres which was sufficient for a measuring time of about 7 hours.

(2) Origin at $t_{\max } / 2$.
4.2 Calorimeter. - Figure 5 shows the calorimeter we used. A stainless steel vacuum can contains the calorimeter and the adiabatic shield. The can is supported from the top of the cryostat by four stainless steel rods and a stainless steel pumping line. The vacuum can is surrounded by liquid helium. Before cooling the vacuum can down it is evacuated to a pressure of $5 \times 10^{-6}$ torr for at least five hours. The twin arrangement is suspended by thin manganin wires $(70 \mu)$, which are thermally anchored to the adiabatic shield. The thermal resistance between the twin arrangement and the shield is greater than $2 \times 10^{5} \mathrm{~K} / \mathrm{W}$.

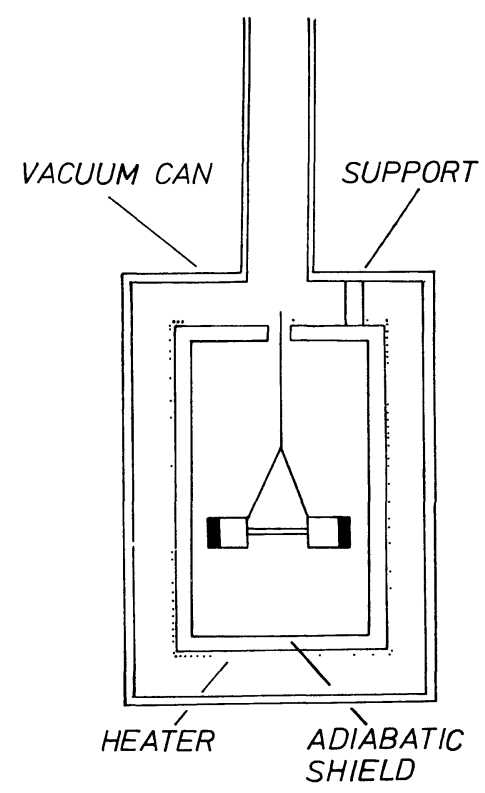

FIG. 5. - Experimental arrangement and calorimeter (schematically).

The heaters are made from $20 \mu$ manganin wire which is wound between two square brass plates (thickness $0.1 \mathrm{~mm}$, edge length $12 \mathrm{~mm}$ ). Improved heaters are in preparation (tungsten plates, thickness $0.03 \mathrm{~mm}$, edge length $14 \mathrm{~mm}$ ). The heater resistances (about 50 ohms) need not be known and since we use a capacitor as energy source it does not matter if the resistances are equal or not. The samples are mounted between the heaters and the other brass plates to which the weak link is soldered (Fig. 1). The thermal resistances between the heaters and the samples need not be equal but they should be as small as possible. A large resistance will increase the relaxation time of the whole arrangement.

4.3. Adiabatic Shield. - The adiabatic shield was machined out of high conductivity copper and supported by a teflon ring from the top of the vacuum can. As heater wire we used manganin with a diameter of $100 \mu$. The power consumption of the shield was about 10 watt maximum. The temperature difference between the twin arrangement and the shield is monitored by a $\mathrm{Au}-0.07$ at \% Fe-chromel thermocouple, 
one junction of which is attached to the shield and the other to the twin arrangement. The output from this thermocouple is taken directly to a high gain dc amplifier used as a null detector. The output of the null detector is fed into a PID temperature controller. It is an important question at which point of the twin arrangement the second junction of the thermocouple is to be mounted. We checked this problem very carefully and decided to solder on each of the inner plates of the sample holder a manganin wire working as a heat delay line. This is important for the following reason. The final temperature difference between the shield and the twin arrangement after heating into heater $\mathrm{H}_{1}$ or heater $\mathrm{H}_{2}$ is in general smaller than the temperature difference between a certain point of the twin arrangement and the shield as long as the equilibrium is not yet reached again. The other junction of the thermocouple is attached to the connected ends of the heat delay lines. The thermal resistance of the heat delay line is about $10^{4} \mathrm{~K} / \mathrm{W}$ and depends slightly on the used sample holder. These precautions guarantee that the temperature difference in question never exceeds $0.1 \mathrm{~K}$ even if the samples are strongly heated. This difference is not critical. Because of the tight coupling of the two specimens we can tolerate large balanced heat leaks due to radiation.

4.4 Thermometry. - We used a silicon temperature sensor (DT-500 P-GR-U, $25 \mathrm{mg}$, Cryophysics) to measure the absolute temperature of the twin arrangement. With this temperature sensor we were able to measure the absolute temperature to an accuracy of $0.5 \mathrm{~K}$ or even better. Due to the special measuring method this accuracy is sufficient. The sensor was mounted on one of the heaters and the temperature was read after reestablishing of equilibrium. To measure the heat flux through the weak link we used uncalibrated thermocouple material since the results do not depend on the thermopower at all. To amplify the $\Delta T(t)$ signal we used a highly stable chopper stabilized pre-amplifier (Zero stability $\pm 1.5 \mathrm{nV} / \mathrm{K}$ ).

The thermopower of the thermocouple is slightly temperature dependent. As a consequence the voltage output is not a linear function of temperature. The deviation, however, is because of the moderate temperature steps small and causes a negligible error (order of magnitude $1 \%$ oo).

5. Experimental procedure. - With the aid of a simple control electronic the apparatus was fully automated. The principal measuring procedure follows from figure 6 .

We cooled the calorimeter by helium exchange gas down to the lowest temperature at which we wanted to start the measurement. Before starting the exchange gas was carefully pulped out. Because of the automation we measured always from lower to higher temperatures. After reaching equilibrium the control electronic and the temperature controller were switched on and the zero point of the pre-amplifier of the temperature controller manipulated in such a manner that the temperature controller saw zero temperature difference between the twin arrangement and the adiabatic shield when the temperature of the shield was slightly lower than the temperature of the twin arrangement $(10 \mathrm{mK})$. In the opposite case the whole arrangement would have become unstable. Without heating the temperature of the twin arrangement drifted very slowly towards lower temperatures. This was due to the wires leading to the samples which were thermally anchored to the shield and due to the radiation. The temperature of the twin arrangement was followed by the temperature of the shield (about $0.5 \mathrm{~K}$ per hour). After switching on the control electronic the following run was repeated again and again :

1) Power input into heater $H_{1}$.

2) Compensating of the temperature difference between shield and twin arrangement (Fig. 6a).

3) Recording of a signal which is proportional to $\Delta T(t)$ in arbitrary voltage units (Fig. $6 b$ ).

4) Recording of a signal which is proportional to

$$
\int_{0}^{t} \Delta T(t) \mathrm{d} t
$$

in arbitrary voltage units (Fig. 6c) $\left(^{3}\right)$.

5) Recording of the absolute temperature $T$ as the voltage drop along the silicion temperature sensor (Fig. 6d).

6) Power input into heater $\mathrm{H}_{2}$ and so on.

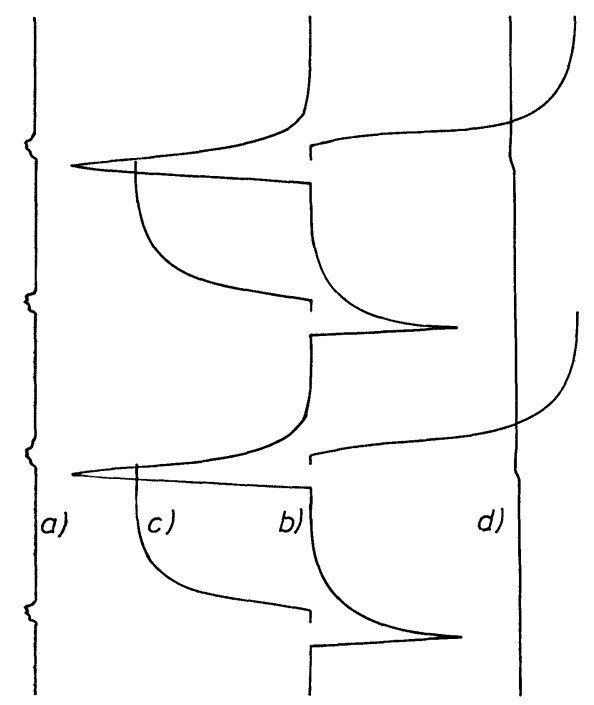

Fig. 6. - Automation of the measuring process : a) temperature difference between adiabatic shield and twin arrangement ; b) temperature difference between the temperature probes; c) signal which is proportional to the integrated heat flux; d) absolute temperature of the twin arrangement.

(3) To integrate electronically to a high degree of accuracy is not an easy task. Therefore (in an improved version of the apparatus) we used a small on-line computer as a digital integrator. 
6. Results. - Figure 7 shows the measured For discussion and interpretation refer to Deenaspecific heat as a function of temperature of $\mathrm{CeAl}_{2}$. das et al. [3]. The weight of the sample was

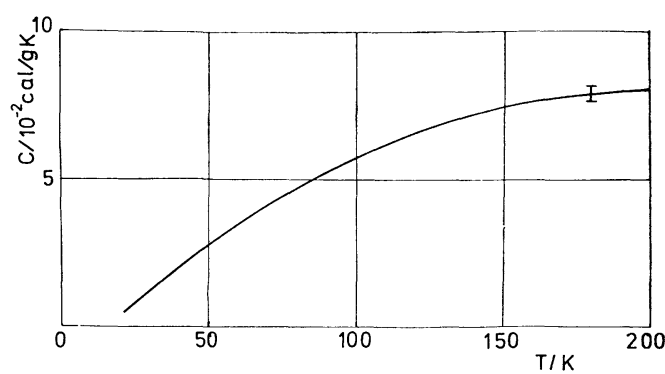

FIG. 7. - Specific heat of $\mathrm{CeAl}_{2}$ as a function of temperature.

\section{$2.5 \mathrm{~g}$.}

Acknowledgments. - The idea of D. Wohlleben to measure specific heat is greatly appreciated. The author thanks K. J. Schmidt for building the highly stable electronic devices, H. Armbrüster, R. Braun, A. Hansen, H. von Löhneysen and R. Pott for many valuable discussions and last not least $F$. Steglich for proof reading, constructive criticism and expert advise.

\section{References}

[1] Jones, R. W., Knapr, G. S., and Veal, B. W., Rev. Sci. Instrum., 44 (1973) 807.

[2] Päsler, M., Phänomenologische Thermodynamik (de Gruyter, Berlin) 1975.

[3] Deenadas, C., Thompson, A. W., Craig, R. S. and WalLACE, W. E., J. Phys. Chem. Solids 32 (1971) 1853. 